\title{
CANTORIAN TABLEAUX REVISITED
}

Michel MEndès FrAnCE

À Jean-Marc Deshouillers, en toute amitié

Abstract: This article is a discussion of Cantor's famous diagonal proof showing the existence of transcendental numbers. We study three questions raised in a previous joint paper [1].

Keywords: transcendental numbers, Cantor's diagonal, continued fractions.

\section{Introduction and definitions}

We start out with a very simple and yet beautiful idea of Cantor's [2] that dates back to 1891.

Fix a basis of numeration $s \geqslant 2$. Consider the infinite tableau $T$ where each row is the $s$-expansion of a real algebraic number in the unit interval $[0,1[$ with the convention that to rational numbers $a / s^{b}$ ( $a>0, b>0$ integers) corresponds two rows, one with a tail $0^{\infty}$ and one with a tail $(s-1)^{\infty}$. Assume all algebraic numbers in $[0,1[$ actually appear in the tableau $T$.

Let

$$
T=\left(\begin{array}{cccc}
a_{11} & a_{12} & a_{13} & \cdots \\
a_{21} & a_{22} & a_{23} & \ldots \\
a_{31} & a_{32} & a_{33} & \cdots \\
\cdots & \cdots & \cdots & \cdots \\
\cdots & \cdots & \cdots & \ldots
\end{array}\right)
$$

where $a_{i j} \in[s]:=\{0,1,2, \ldots, s-1\}$. Cantor's goal was to establish the existence of transcendental numbers. He argued as follows. For every $n$, define $b_{n} \neq a_{n n}, b_{n} \in$ $[s]$. Then obviously the number

$$
b=. b_{1} b_{2} b_{3} \cdots
$$

cannot be a row of $T$ since if it where, then it would appear at some rank say $i$, and we would conclude that $b_{i}=a_{i i}$ which is absurd. Therefore the number $b$ is transcendental. 
In [1] we actually show that the diagonal number

$$
a=. a_{11} a_{22} a_{33} \cdots
$$

is itself transcendental; no need to perturb the diagonal. The proof goes as follows. First observe that to each algebraic number

$$
\alpha_{i}=. a_{i 1} a_{i 2} \cdots a_{i n} \cdots
$$

corresponds at least one algebraic number

$$
\alpha_{k}=. a_{k 1} a_{k 2} \cdots a_{k n} \cdots
$$

such that for all $n, a_{k n} \neq a_{i n}$. For example, in basis $2, \alpha_{k}=1-\alpha_{i}$ and in basis $s \geqslant 3, \alpha_{k}=\alpha_{i}+1 /(s-1)(\bmod 1)$.

Now if $a$ was algebraic, it would appear at some row $i$ of the tableau $T$, so $a=\alpha_{i}$. Let $\alpha_{k}$ as above. Then $a_{k k}$ is the $k$-th digit of $\alpha_{i}$ and therefore $a_{k k}=a_{i k}$, which contradicts $a_{k n} \neq a_{i n}$ for $n=k$. This establishes the transcendency of $a$.

Permuting the rows of $T$ creates new diagonals and hence new transcendental numbers. Define the permanent of $T, P(T)$, to be the set of all the diagonals when permuting the rows in all possible ways. In [1] we showed that for $s=2, P(T)$ is the set of all transcendental numbers in the unit interval. Actually R. Gray [3] established before us a similar result concerning the disturbed diagonals but clearly for $s=2$ this is really the same result as ours!

Now for $s \geqslant 3$ the situation is quite different. In [1] we established that the transcendental numbers in $P(T)$ contain each infinitely many times each one of the $s$-digits $0,1,2, \ldots, s-1$. Liouville transcendental numbers

$$
\sum_{n=0}^{\infty} 1 / s^{n !}
$$

are therefore missing.

Let $V$ be the family of those transcendental numbers which each contain infinitely many times each one of the digits $0,1,2, \ldots, s-1$. The question arises whether $P(T)=V$ or $P(T) \varsubsetneqq V$. We shall prove here (Theorem 3 ) that it is the second possibility that holds.

We now extend our definitions. $T$ will be a finite or infinite square tableau on an alphabet $A=\left\{a_{1}, a_{2}, \ldots, a_{s}\right\}$ which at the very end of our paper, may well become infinite. Let $L(T)$ be the set of words (resp. sequences) formed by the rows. The permanent $P(T)$ is as before the set of the diagonals of $T$ and of all the tableaux obtained by permuting the rows. In Cantor's example above, $L(T)$ is the family of algebraic numbers (or rather their $s$-expansions) in $[0,1[$ and $P(T)$ is a subset of transcendental numbers. Then of course $L(T) \cap P(T)=\emptyset$. 
In the general case we agree to say that $T$ is a Cantorian tableau if $L(T) \cap$ $P(T)=\emptyset$. A theorem becomes a definition. Here are a few examples. Choose $A=\{a, b\}$. Easily seen, the two tableaux

$$
\left(\begin{array}{ll}
a & b \\
b & a
\end{array}\right) \quad\left(\begin{array}{lll}
a & b & a \\
b & a & b \\
b & a & b
\end{array}\right)
$$

are Cantorian. In the second one the two last rows are equal; this is not prohibited. The next tableau

$$
\left(\begin{array}{lll}
a & b & a \\
a & a & a \\
b & b & b
\end{array}\right)
$$

is not Cantorian since $L(T) \cap P(T) \ni a b a$ (permute rows 2 and 3 ).

Denote by $C(n, s)$ the number of Cantorian tableaux of size $n \times n$ on an alphabet with $s$ letters. Obviously

$$
C(n, s) \leqslant s^{n^{2}}
$$

Estimating $C(n, s)$ seems in general quite difficult. We shall show (Theorems 1 and 2) that

$$
C(2, s)=s^{2}(s-1)^{2},
$$

and that, as $n$ grows to infinity,

$$
C(n, 2) \geqslant 2^{\{.61+o(1)\} n^{2}} .
$$

The inequality improves a result of [1] where we had shown the exponent to be bounded by below by $\left\{\frac{1}{2}+o(1)\right\} n^{2}$.

It would obviously be interesting to know the value of

$$
\lim _{n \rightarrow \infty} \log C(n, 2) / \log 2^{n^{2}}
$$

if it exists. Our result shows that the corresponding liminf is greater than $.61 \ldots$ (actually we'll show that the lower bound is larger than .6169). Comparing with numerical evidence leads to the conjecture

$$
\begin{array}{r|ccccccccccc}
\lim _{n \rightarrow \infty} \frac{\log C(n, 2)}{\log 2^{n^{2}}}=.6169 \ldots \\
\frac{n}{n} & 2 & 3 & 4 & 5 & 6 & 7 & 8 & 9 & 10 & 11 \\
\hline 10^{3} \frac{\log C(n, 2)}{\log 2^{n^{2}}} & 500 & 509 & 673 & 657 & 675 & 656 & 651 & 632 & 626 & 610
\end{array}
$$




\section{The value of $C(2, s)$}

Theorem 1. The number of $2 \times 2$ Cantorian tableaux on an $s$ letter alphabet is

$$
C(2, s)=s^{2}(s-1)^{2}
$$

Proof. The theorem is a consequence of a slightly more general result. Let $N(n, s)$ be the number of non-Cantorian tableaux of size $n \times n$. Then $C(n, s)+N(n, s)=$ $s^{n^{2}}$. Let $M(n, s)$ be the number of $n \times n$ tableaux $T$ such that the diagonal of $T$ appears among the rows. Obviously

$$
M(n, s) \leqslant N(n, s) .
$$

$M(n, s)$ is easily computed. Indeed, first note that the number of tableaux such that row 1 and the diagonal coincide is $s^{n^{2}-n+1}$. Row 1 plays no special role so that the number of tableaux $T$ such that row $i=$ diagonal is also $2^{n^{2}-n+1}$. In terms of probability, denoting by $A_{i}$ the event that row $i$ is equal to the diagonal, we thus obtain

$$
\operatorname{Prob}\left(A_{i}\right)=\frac{s^{n^{2}-n+1}}{s^{n^{2}}}=\frac{1}{s^{n-1}} .
$$

Now, it is easy to see that the events $A_{i}$ are independent. Therefore

$$
\begin{aligned}
\operatorname{Prob}\left(\cup_{1 \leqslant i \leqslant n} A_{i}\right) & =\left(\begin{array}{c}
n \\
1
\end{array}\right) \frac{1}{s^{n-1}}-\left(\begin{array}{l}
n \\
2
\end{array}\right)\left(\frac{1}{s^{n-1}}\right)^{2}+\left(\begin{array}{l}
n \\
3
\end{array}\right)\left(\frac{1}{s^{n-1}}\right)^{3}-\cdots \\
& =1-\left(1-\frac{1}{s^{n-1}}\right)^{n} .
\end{aligned}
$$

Therefore

$$
M(n, s)=s^{n^{2}}\left[1-\left(1-\frac{1}{s^{n-1}}\right)^{n}\right]=s^{n^{2}}-\left(s^{n}-s\right)^{n} .
$$

Unfortunately the inequality $N(n, s) \geqslant M(n, s)$ does not convey much information on the size of $N(n, s)$ as $n \rightarrow \infty$.

We now specialize to $n=2$ :

$$
M(2, s)=s^{4}-\left(s^{2}-s\right)^{2}
$$

In this very particular case, observe that

$$
N(2, s)=M(2, s)
$$

so that $C(2, s)=s^{4}-M(2, s)=\left(s^{2}-s\right)^{2}$. 


\section{A lower bound for $C(n, 2)$}

\section{Theorem 2.}

$$
\lim _{n \rightarrow \infty} \inf \frac{\log C(n, 2)}{\log 2^{n^{2}}} \geqslant .6169486436 \ldots
$$

Proof. Let $3 \leqslant k \leqslant n$. Consider the following $n \times n$ tableau $T$ on the alphabet $A=\{a, b\}$.

- Row 1 contains only $b$ 's.

- Row 2 , row $3, \ldots$, row $k-1$ contain each $k$ occurrences of $b$.

- The last $n-k+1$ rows contain only $a$ 's.

Such a tableau is necessarily Cantorian since the number of occurrences of $b$ in each word of the permanent $P(T)$ is at least 1 and at most $k-1$. Therefore $P(T) \cap L(T)=\emptyset$.

Now count the number of such $T$ for a given $k$. For each row $2,3, \ldots, k-1$ there are $\left(\begin{array}{l}n \\ k\end{array}\right)$ possibilities, hence $\left(\begin{array}{l}n \\ k\end{array}\right)^{k-2}$ possible tableaux. Therefore

$$
C(n, 2) \geqslant \max _{k}\left(\begin{array}{l}
n \\
k
\end{array}\right)^{k-2} \text {. }
$$

To approximate this lower bound, put $k=\alpha n, 0<\alpha<1$. As $n \rightarrow \infty$, we have

$$
\frac{\log \left(\begin{array}{c}
n \\
\alpha n
\end{array}\right)^{\alpha n-2}}{\log 2^{n^{2}}} \rightarrow \phi(\alpha)
$$

where

$$
\phi(\alpha)=\left(\alpha^{2} \log \frac{1}{\alpha}+\alpha(1-\alpha) \log \frac{1}{1-\alpha}\right) / \log 2 .
$$

Numerical computation shows that

$$
\max _{0<\alpha<1} \phi(\alpha) \approx .6169486436
$$

At this point one may justly ask what can be said about $C(n, s)$ for $s>2$. In Section 1, we mentioned the following property. A sufficient condition for a tableau, be it finite or not, to be Cantorian is that to each row corresponds at least one row which differs from it coordinate-wise. As a consequence, it is easy to see that

$$
C(n, s) \geqslant\{s(s-1)\}^{n[n / 2]}
$$

and therefore

$$
\liminf _{n \rightarrow \infty} \frac{\log C(n, s)}{\log \left(s^{n^{2}}\right)} \geqslant \frac{1}{2}\left(1+\frac{\log (s-1)}{\log s}\right) .
$$

For $s=2$, this lower bound is not as good as our Theorem 2. Note however that it tends to 1 as $s$ increases to infinity. 


\section{Infinite tableaux}

We restrict ourselves to the most striking case namely when the rows are the $s$-expansions of the algebraic numbers in the unit interval. We know that $P(T)$ represents the $s$-expansions of transcendental numbers. Let $V$ be those transcendental numbers whose $s$-expansion contains infinitely often each one of the $s$-digits $0,1, \ldots, s-1$. For $s=2, \mathrm{~V}$ is the family of all the transcendental numbers in $[0,1[$, and we already mentioned that in that case $P(T)=V$ (see [1] and $[3])$. If $s \geqslant 3$ we also know that $P(T) \subset V$ (see [1]).

Theorem 3. Suppose $s \geqslant 3$. Then

$$
P(T) \varsubsetneqq V
$$

Proof. Let $x \in[0,1[$

$$
x=. x_{1} x_{2} x_{3} \cdots \quad x_{j} \in[s] .
$$

We define the shell of $x$ to be the set $\sigma(x)$ of numbers

$$
y=. y_{1} y_{2} y_{3} \cdots \quad y_{j} \in[s]
$$

such that for all $j, y_{j} \neq x_{j}$. Obviously $y \in \sigma(x) \Leftrightarrow x \in \sigma(y)$. Numbers should be associated to their $s$-expansion and conversely so that those rationals with two different expansions should be considered as distinct numbers.

Cantor's initial argument using perturbed diagonals states that if

$$
d=. a_{11} a_{22} a_{33} \cdots
$$

is the diagonal of $T$, then $\sigma(d)$ consists of transcendental numbers and more generally, $\sigma(P(T))$ also.

Let $x$ be any algebraic number the $s$-expansion of which contains at least two digits each one infinitely often, for example $x=\frac{s}{s^{2}-1}$. Consider the set $\sigma(x)$.

Since $s \geqslant 3, \sigma(x)$ is uncountable and sufficiently large so that $\sigma(x) \cap V \neq \emptyset$.

We claim that $P(T) \cap \sigma(x) \cap V=\emptyset$. If this is true then there exists $z \in V$ (actually $z \in V \cap \sigma(x))$ such that $z \notin P(T)$. As a matter of fact all numbers in $V \cap \sigma(x)$ would share that property. The theorem would then be established.

So we are left to prove $P(T) \cap \sigma(x) \cap V=\emptyset$. Suppose that on the contrary $P(T) \cap \sigma(x) \cap V \neq \emptyset$. Let $y \in P(T) \cap \sigma(x) \cap V$. In particular $y \in P(T)$ and by Cantor's argument $\sigma(y)$ only contains transcendental numbers. On the other hand $y \in \sigma(x)$ so that $x \in \sigma(y)$ this is absurd; since $x$ is algebraic. 


\section{A final remark}

Instead of $s$-expansions one could consider for example continued fraction expansions of real irrational numbers in $[0,1[$.

$$
\left[0, a_{1}, a_{2}, a_{3}, \cdots\right]
$$

The partial quotients $a_{j}$ belong to $\mathbb{N}=\{1,2,3, \ldots\}$ so the alphabet we now consider is infinite. Suppose we wished to mimic some of our previous results. We would consider an infinite tableau each row being the sequence of partial quotients of an irrational algebraic number in ]0,1[. If we were to prove that the diagonal of this tableau represents the continued fraction expansion of a transcendental number, we would need the following unknown (?) property:

To each algebraic irrational number

$$
\alpha=\left[0, a_{1}, a_{2}, a_{3}, \cdots\right]
$$

corresponds at least one algebraic irrational number $\beta$

$$
\beta=\left[0, b_{1}, b_{2}, b_{3}, \cdots\right]
$$

such that for all $i, b_{i} \neq a_{i}$.

This is obviously true if $\alpha$ is quadratic ... but this is the only case I know of. Allow me to call this a conjecture.

Curiously enough this is trivial in another context. Let $k$ be a finite field with characteristic $p \neq 0 . k\left(\left(X^{-1}\right)\right)$ represents the field of Laurent series with coefficients in $k$. Elements $\alpha(X) \in k\left(\left(X^{-1}\right)\right)$ have continued fraction expansions

$$
\alpha(X)=\left[a_{0}(X), a_{1}(X), a_{2}(X), \cdots\right]
$$

where the partial quotients are polynomials and $j \geqslant 1 \Rightarrow$ degree $a_{j}(X) \geqslant 1$.

Algebraic elements in $k\left(\left(X^{-1}\right)\right)$ are naturally defined. Let $T$ be a tableau whose rows are the continued expansions of the algebraic irrational elements $\alpha(X)$ "in the unit interval" i.e. for which $a_{0}(X)=0$.

Claim. The diagonal of $T$ is the continued fraction expansion of a transcendental elements in $k\left(\left(X^{-1}\right)\right)$.

Proof. To every algebraic element

$$
\alpha(X)=\left[0, a_{1}(X), a_{2}(X), \cdots\right]
$$

corresponds an algebraic element

$$
\beta(X)=\alpha\left(X^{p}\right)=\left[0, a_{1}\left(X^{p}\right), a_{2}\left(X^{p}\right), \cdots\right]
$$

and trivially, $\forall i, a_{i}\left(X^{p}\right) \neq a_{i}(X)$. Then conclude as in Section 1.

L'auteur tient remercier Jean Creignou et Gérald Tenenbaum pour leur aide. 


\section{References}

[1] S. Brlek, M. Mendès France, J.M. Robson, M. Rubey, Cantorian Tableaux and Permanents, L'Enseignement Mathématique, vol. 50, 2004, pp. 524-542.

[2] G. Cantor, Über eine elementare Frage der Mannigfaltigkeitslehre, Jahresbericht der Deutschen Mathematiker-Vereinigung, vol. 1 ,1891, pp. 75-78; Gesammelte Abhandlungen, Springer 1932, pp. 278-281.

[3] R. Gray, George Cantor and Transcendental Numbers, Amer. Math. Monthly, vol. 101, 1994, pp. 819-832.

Address: Institut de Mathématiques de Bordeaux, UMR 5465, Université Bordeaux 1, F-33405 Talence cedex, France

E-mail: michel.mendes-france $\varrho_{w}$ anadoo.fr

Received: 5 December 2006; revised: 26 January 2007 